\title{
High average and peak power femtosecond large-pitch photonic-crystal-fiber laser
}

\author{
Martin Baumgartll, ${ }^{1,2, *}$ Florian Jansen, ${ }^{1}$ Fabian Stutzki, ${ }^{1}$ Cesar Jauregui, ${ }^{1}$ Bülend Ortaç, ${ }^{3}$ \\ Jens Limpert, ${ }^{1,2}$ and Andreas Tünnermann ${ }^{1,2,4}$ \\ ${ }^{1}$ Friedrich-Schiller-University Jena, Institute of Applied Physics, Albert-Einstein-Strasse 15, 07745 Jena, Germany \\ ${ }^{2}$ Helmholtz-Institute Jena, Max-Wien-Platz 1, 07743 Jena, Germany \\ ${ }^{3}$ UNAM-Institute of Materials Science and Nanotechnology, Bilkent University, 06800 Bilkent, Ankara, Turkey \\ ${ }^{4}$ Fraunhofer Institute for Applied Optics and Precision Engineering, Albert-Einstein-Strasse 7, 07745 Jena, Germany \\ ${ }^{*}$ Corresponding author: martin.baumgart@@uni-jena.de
}

Received October 26, 2010; revised December 10, 2010; accepted December 17, 2010; posted December 22, 2010 (Doc. ID 137235); published January 12, 2011

We report on the generation of high-average-power and high-peak-power ultrashort pulses from a mode-locked fiber laser operating in the all-normal-dispersion regime. As gain medium, a large-mode-area ytterbium-doped large-pitch photonic-crystal fiber is used. The self-starting fiber laser delivers $27 \mathrm{~W}$ of average power at $50.57 \mathrm{MHz}$ repetition rate, resulting in $534 \mathrm{~nJ}$ of pulse energy. The laser produces positively chirped $2 \mathrm{ps}$ output pulses, which are compressed down to sub-100 fs, leading to pulse peak powers as high as 3.2 MW. (c) 2011 Optical Society of America OCIS codes: $\quad 060.2310,060.2320,140.3510,140.4050,140.3615,140.7090$.

The development of powerful ultrashort pulse laser sources opens a door to the study and understanding of fundamental effects in nature. High-average powers combined with high pulse peak-powers are required to initiate nonlinear processes and allow for a reasonable detection procedure. A new generation of laser oscillators with the highest-average-power levels to date have been demonstrated using $\mathrm{Yb}$-doped thin-disk technology in efficient combination with ultrafast saturable absorption mechanisms [1,2]. However, the ultrashort pulse generation from this active medium is limited, due to the relatively narrow emission bandwidth of common crystalline materials. On the other hand, Yb-doped optical fibers possess promising properties with very large emission bandwidths and high single-pass gains. The laser performance in terms of average power and energy extraction is limited mainly by the excessive nonlinearity of the fibers. Significant progress concerning the performance of mode-locked fiber lasers in terms of pulse duration and energy has been enabled by the development of new propagation regimes to overcome the limitations imposed by nonlinearities. The most powerful mode-locked fiber lasers have been developed in the all-normaldispersion regime. Such systems, supporting dissipative soliton pulse evolution, have been able to produce sub$100 \mathrm{fs}$ pulses with energies as high as $31 \mathrm{~nJ}$ using standard single-mode fibers [3]. More recently, exceptional performances in terms of pulse energy and peak power have been demonstrated in mode-locked fiber lasers by overcoming nonlinear effects using large-mode-area (LMA) photonic-crystal fibers (PCF) [늠]. Most of these systems use a high modulation-depth semiconductorbased saturable absorber mirror (SAM) for nonlinear self-amplitude modulation. This limits average-power scaling owing to high nonsaturable losses of the absorber and low thermal conductivity of the substrate, which leads to a strong thermal load and to degradation of the SAM. Another approach is the use of a narrow spectral filter to achieve strong self-amplitude modulation inside the laser cavity [7].

0146-9592/11/020244-03\$15.00/0
In this Letter, we report the generation of femtosecond pulses from an all-normal-dispersion fiber laser featuring a large-mode-area ytterbium-doped large-pitch photoniccrystal fiber. The combination of an instantaneous, nonabsorptive mode-locking mechanism [nonlinear polarization rotation (NPR) technique] with a novel PCF design in a spectral-filtering effect assisted chirped-pulse oscillator configuration enables a significant peak power enhancement with the generation of multimegawatt pulses from a fiber laser. The laser reaches $27 \mathrm{~W}$ of average power at a $50.57 \mathrm{MHz}$ repetition rate, corresponding to $534 \mathrm{~nJ}$ pulses. The output pulses are extracavity dechirped down to 100 fs with $3.2 \mathrm{MW}$ peak power. To the best of our knowledge, these are the highest average and peak powers ever reached by a mode-locked fiber laser.

The high-power passively mode-locked fiber laser is set up in a ring-cavity configuration, as shown in Fig. 1. The gain fiber was designed on the basis of numerical simulations [8]. Different structures with variable numbers of air holes, pitch, and hole sizes were simulated in order to find the optimized set of parameters for robust single-mode operation. The fiber has a hexagonal lattice

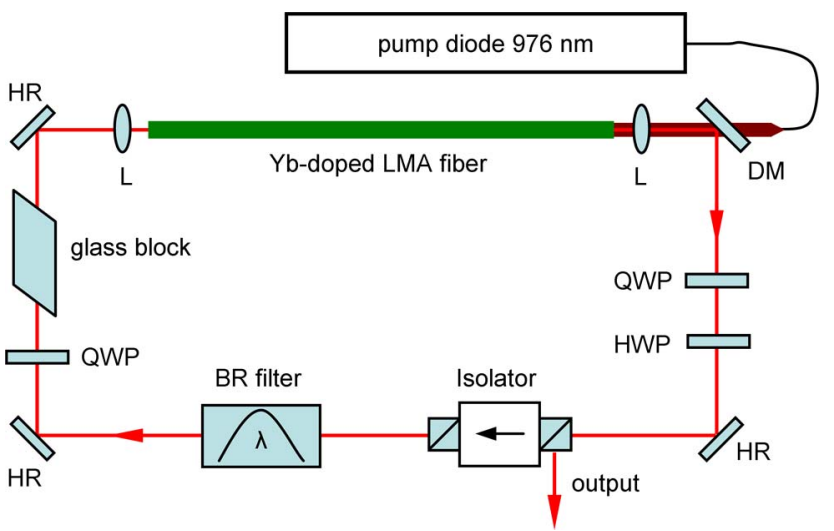

Fig. 1. (Color online) Schematic set-up. L, lens; DM, dichroic mirror; HR, high reflection mirror; QWP and HWP, quarter- and half-wave plate; BR filter, birefringent filter. 

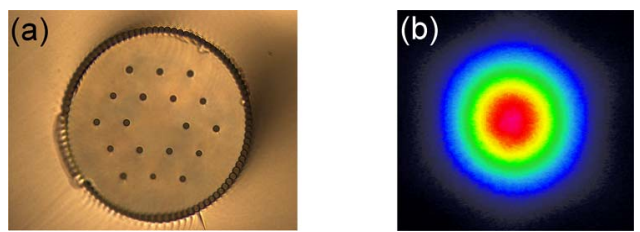

Fig. 2. (Color online) (a) Microscope image of the fiber cross section. (b) Beam profile at $27 \mathrm{~W}$ output power.

of air holes, wherein the core is formed by one missing hole in the center [Fig. 2(a)]. The core is surrounded by two rings of air holes. An air hole diameter of $10 \mu \mathrm{m}$ and a large pitch of $30 \mu \mathrm{m}$ between them results in a mode field diameter of $41 \mu \mathrm{m}$ for the fundamental mode at $1030 \mathrm{~nm}$ wavelength. Hence, the fiber exhibits strongly reduced light intensities in the core while maintaining effectively single-mode behavior. Therefore, in spite of the large core of the fiber, the presented laser does not require any additional mode-filter, unlike that presented in [4]. A $170 \mu \mathrm{m}$ air cladding together with a high doping concentration provides efficient pump light absorption $(24 \mathrm{~dB} / \mathrm{m}$ small signal absorption at $976 \mathrm{~nm})$. A fiber length of $1.2 \mathrm{~m}$ is chosen to obtain sufficient gain. In the experiment, the fiber end faces are angled $\left(6^{\circ}\right)$ to suppress back reflections. A polarizing optical isolator ensures unidirectional operation, and its first polarizer serves as rejection port for the NPR mode-locking technique. This rejection port is used as laser output at the same time. A set of one half- and two quarter-wave plates is used to control the output coupling ratio and the NPR in the fiber. To support dissipative soliton stability, a birefringent filter with a $16 \mathrm{~nm}$ FWHM bandwidth is employed, resulting in further self-amplitude modulation. Behind the filter, a block of $30 \mathrm{~cm}$ bulk fused silica is placed in Brewster angle into the remaining free-space section, providing a small amount of prestretching before the pulse enters the fiber. The total cavity group-delay dispersion of the all-normal-dispersion setup, including all bulk optical elements, is estimated to be $\sim 0.03 \mathrm{ps}^{2}$. The beam profile is monitored and the fiber yields an excellent beam quality $\left(M^{2}=1.2\right)$, as indicated by the profile [Fig. 2(b)] measured in the high-power regime.

An optimization of the setting of the wave-plates results in stable mode-locked operation. The mode-locking threshold for this wave plate setting is reached at a pump power of more than $40 \mathrm{~W}$. Increase of the pump to more than $52 \mathrm{~W}$ leads to break up of cw single-pulse modelocking. A sudden power drop to $\sim 60 \%$ of the output power is observed when the wave plates are rotated to switch the laser to $\mathrm{cw}$ operation, which is a sign of the strong influence of NPR. Without the filter, mode-locked states are hardly obtained, the operation is less robust, and the tendency to generate multipulsing states is increased. The spectral filter plays an important role for pulse shaping in this laser, as discussed in [9]. In contrast, by use of a very narrow spectral filter, mode locking is obtained easily for various wave plate settings, but for most of these cases, more than one pulse is circulating in the cavity. The relatively broad $16 \mathrm{~nm}$ filter is found to be an optimum. The highest output power for fundamental mode locking is obtained at a pump power of $\sim 52 \mathrm{~W}$. With this parameter setting the laser operates in a self-starting single-pulse regime at a repetition rate of $50.57 \mathrm{MHz}$. The
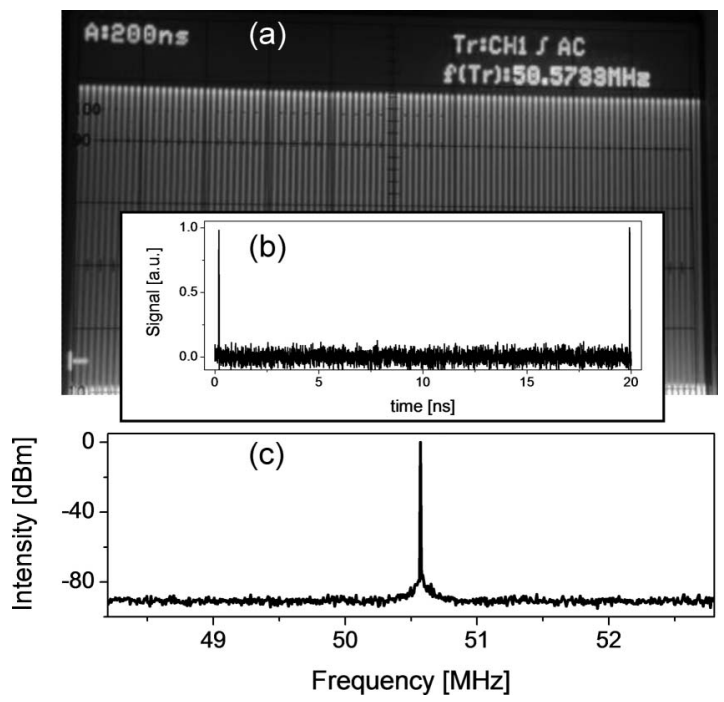

Fig. 3. (a) Pulse train on analog oscilloscope, (b) two consecutive peaks on a $50 \mathrm{GHz}$ sampling oscilloscope (resolution 5 ps), (c) rf spectrum, resolution bandwidth $300 \mathrm{~Hz}$.

pulse train is monitored with a $200 \mathrm{MHz}$ analogue oscilloscope and a $50 \mathrm{GHz}$ sampling oscilloscope in combination with an $18.5 \mathrm{ps}$ response time photodiode to ensure single-pulse operation (Fig. 3). Furthermore, a long scan range (150 ps) autocorrelator is used. The oscilloscope trace [Fig. 3(a)] shows high amplitude stability. This is confirmed by an rf spectrum analyzer measurement [Fig. 3(c)], which exhibits more than $80 \mathrm{~dB}$ separation between the fundamental harmonic and the background.

The laser delivers spectrally broad output pulses with a full spectral width at half-maximum of $32.5 \mathrm{~nm}$ at a central wavelength of $1032.4 \mathrm{~nm}$. The spectrum is shown in Fig. 4. It possesses a steep-edged shape due to the strong influence of self-phase modulation on pulse shaping. The inset of Fig. 5 shows the autocorrelation trace measured directly at the laser output. The positively chirped pulses yield an autocorrelation width (FWHM) of $2.4 \mathrm{ps}$. The trace exhibits a triangle-like shape, indicating that the stretched pulses mimic the quasi-rectangular spectral shape in the temporal domain. Using a high-efficiency transmission grating pair with 1250 lines $/ \mathrm{mm}$, these pulses are compressed externally to a minimum autocorrelation width of $134 \mathrm{fs}$ (Fig. 5), which corresponds to a compression factor of 18 . The dispersion provided by the grating compressor is about $-0.036 \mathrm{ps}^{2}$. With a deconvolution factor of 0.74 , which is calculated from the power

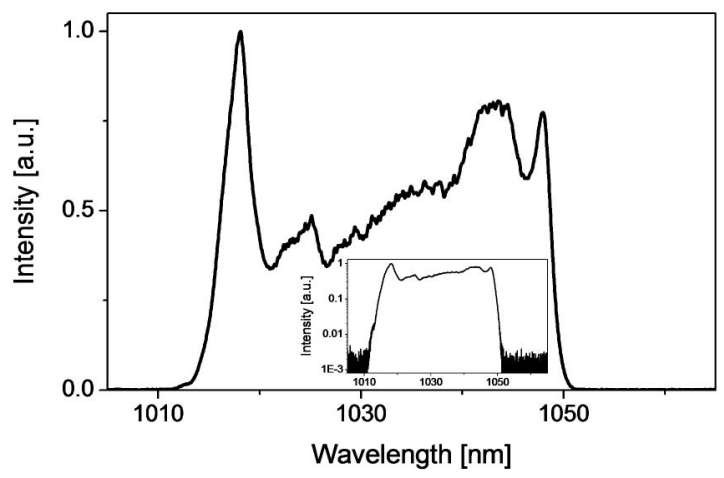

Fig. 4. Optical spectrum; inset, logarithmic scale. 


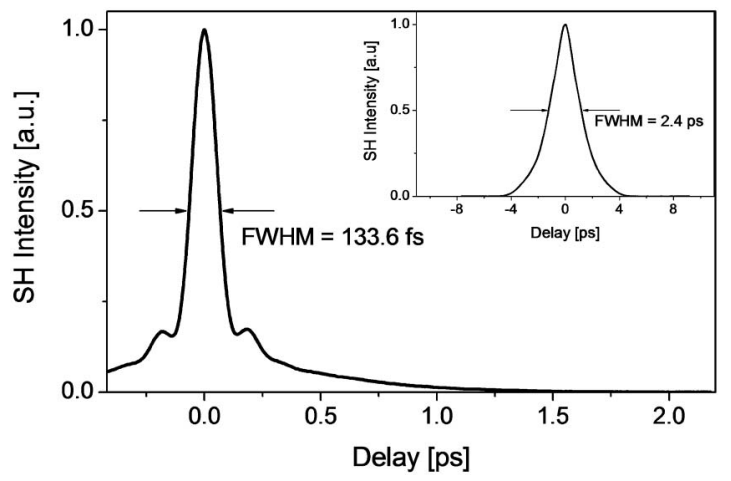

Fig. 5. Autocorrelation traces, externally compressed pulse; inset, chirped pulse.

spectrum, the compressed pulse duration computes to a value of $98 \mathrm{fs}$. Hence, the duration is $20 \%$ above the transform limit, which implies that the compressed pulses possess a residual higher-order phase, which cannot be compensated by the grating compressor. Because of the aforementioned complex temporal shape of the stretched pulses, they accumulate a certain amount of higher-order phase due to Kerr nonlinearity in the fiber. In this regime with only $52 \mathrm{~W}$ of pump power an average power of $27 \mathrm{~W}$ is measured at the output of the laser, yielding an optical-to-optical efficiency well above $50 \%$. This corresponds to pulse energies of $534 \mathrm{~nJ}$. Hence, the obtained pulse energy relative to the mode area of the fiber is comparable with the highest performance achieved with standard single-mode all-normaldispersion fiber lasers [3]. The output coupling ratio is estimated to be $\sim 80 \%$. The autocorrelation trace of the compressed pulses is shown in Fig. 5. It exhibits a pedestal, which results both from the steep spectral shape and from the above-mentioned uncompensated higherorder phase. The amount of energy contained inside the main pulse is estimated to be $\sim 75 \%$. An additional loss (20\%) results from the grating compressor efficiency, yielding $427 \mathrm{~nJ}$ of compressed pulse energy. This, consequently, results in a peak power of more than $3.2 \mathrm{MW}$, which is, to our knowledge, the highest peak power generated by a mode-locked fiber laser.
In conclusion, we have presented a passively modelocked Yb-doped fiber laser based on a large-pitch photonic-crystal fiber. The unprecedented performance is enabled by the use of a very large-mode-area fiber that exhibits strongly decreased nonlinearity in combination with a nonabsorptive instantaneous mode-locking mechanism and a spectral filter element. A relatively short fiber length and an additional amount of bulk dispersion enables the generation of broadband pulses with $27 \mathrm{~W}$ of record average power corresponding to $534 \mathrm{~nJ}$ of energy, which could be compressed to a duration of $100 \mathrm{fs}$. Hence, peak powers exceeding 3.2 MW are obtained from a fiber.

We acknowledge support by the German Federal Ministry of Education and Research (BMBF) under contract 13N10773 and the Inter Carnot \& Fraunhofer Program under project APUS.

\section{References}

1. J. Neuhaus, D. Bauer, J. Zhang, A. Killi, J. Kleinbauer, M. Kumkar, S. Weiler, M. Guina, D. H. Sutter, and T. Dekorsy, Opt. Express 16, 20530 (2008).

2. C. R. E. Baer, C. Kränkel, C. J. Saraceno, O. H. Heckl, M. Golling, R. Peters, K. Petermann, T. Südmeyer, G. Huber, and U. Keller, Opt. Lett. 35, 2302 (2010).

3. K. Kieu, W. Renninger, A. Chong, and F. Wise, Opt. Lett. 34, 593 (2009).

4. B. Ortaç, M. Baumgartl, J. Limpert, and A. Tünnermann, Opt. Lett. 34, 1585 (2009).

5. M. Baumgartl, B. Ortaç, C. Lecaplain, A. Hideur, J. Limpert, and A. Tünnermann, Opt. Lett. 35, 2311 (2010).

6. C. Lecaplain, B. Ortaç, G. Machinet, J. Boullet, M. Baumgartl, T. Schreiber, E. Cormier, and A. Hideur, Opt. Lett. 35, 3156 (2010).

7. S. Lefrançois, K. Kieu, Y. Deng, J. D. Kafka, and F. W. Wise, Opt. Lett. 35, 1569 (2010).

8. F. Jansen, F. Stutzki, H. Otto, M. Baumgartl, C. Jauregui, J. Limpert, and A. Tünnermann, Opt. Express 18, 26834 (2010).

9. A. Chong, W. Renninger, and F. Wise, J. Opt. Soc. Am. B 25 , 140 (2008). 